\title{
TRANSPORT MECHANISMS IN WATER
}

\author{
A. M. AITSAM \\ Baltic Sea Department of the Institute of Thermophysics and Electrophysics, \\ Academy of Sciences of the Estonian SSR, Tallinn, USSR
}

\begin{abstract}
The transport mechanisms of substances in natural water bodies are considered. Dissolved substances can be regarded as passive in that they do not affect the velocity field of the water bodies. The particles of substances can be regarded as either passive or active. Transport of substances is caused by convection, and/or molecular and turbulent diffusion. The buoyancy forces thus have some influence on the transport of substances vertically. As a rule the vertical transport of substances is several orders of magnitude less than the horizontal. One can usually neglect molecular diffusion. Convection transports the substances as solid body. The substance spreads over the whole water body by diffusion; thus the plume edges are relatively sharp. Horizontal diffusion depends mainly on the scale of the various processes and vertical diffusion on scale and fluid stratification. In stable stratified water bodies, vertical diffusion is much reduced and sometimes becomes of the same order as molecular diffusion. The horizontal and vertical diffusions are of great importance in rivers and in the upper and bottom Ekman layers of oceans, seas and lakes. The thermocline and halocline are practically impenetrable for vertical turbulent transport. Vertical flows through them appear to be due to double-diffusivity convection due to the overturning of internal waves and due to upwelling water in the coastal areas.
\end{abstract}

\section{INTRODUCTION}

Substances can be divided into two categories according to the mechanism of their transport in water bodies, whether they are dissolved or not. The former are mixed with water on the molecular scale and are in practice transported by water without any 'slipping' of particles relative to water molecules. Thus the internal forces act on the particles throughout the water body. The non-dissolved substances (suspensions and colloidal solutions) are mixed with water on a scale much larger than molecular. That is why the particles of non-dissolved substances can 'slip' relative to water molecules, i.e. the action of the internal forces must be regarded separately on particles and water. In this case an interaction of forces between water and non-dissolved particles takes place.

As a rule the dissolved substances may be regarded as passive, i.e. they do not affect the structure of water movement. The non-dissolved substances are non-passive. Thus, if suspensions and colloidal solutions are present in water, the water movement cannot then be regarded separately from the movement of non-dissolved particles. 


\section{A. M. AITSAM}

This paper deals mainly with the principles of the transport mechanisms of substances in natural water bodies under laminar and turbulent flow régimes.

\section{TRANSPORT MECHANISM IN LAMINAR FLOW}

It is well known that if in one part of a water body the concentration of some substance is higher than in other parts, then in the long run the concentration will level off over the whole water body. Thus, substances will flow from the higher concentration regions to the lower concentration regions. In standing water the levelling-off of the concentrations of substances takes place by thermal movement of substances and water molecules, and the process is known as molecular diffusion. In molecular diffusion the substance is transferred by the random motion of molecules down a concentration gradient without any over-all transport of fluid taking place. According to Nernst, the rate of flow of substance through unit area perpendicular to the direction $\mathrm{OX}$ is

$$
q_{x}=-D \partial S / \partial x
$$

where $D$ is the coefficient of diffusion and $S$ is the concentration of the substance. In the more general case the rate of flow of substance is

$$
\vec{q}=-D \operatorname{grad} S
$$

where the arrow denotes a vector quantity.

On the basis of statistical physics one can derive the relationship of the molecular diffusion coefficient to the state parameters of both water and substances as follows ${ }^{1}$

$$
D=\mu k T
$$

where $T$ is the absolute temperature, $k$ is a universal constant and $\mu$ is a travelling coefficient.

The total rate of flow of dissolved substance in laminar water motion will be equal to

$$
\vec{q}=-D \operatorname{grad} S+\vec{v} S
$$

where $\vec{v}$ is the water velocity vector.

The corresponding mass balance equation can then be written

$$
\partial S / \partial t+u_{\alpha}\left(\partial S / \partial x_{\alpha}\right)=D \Delta S
$$

where $\Delta$ is the Laplace operator, assumed to summarize after twice repeating indexes.

It can be shown that the mass balance equation given is a parabolic one, e.g. the substance spreads from an instantaneous point source at zero time over the whole water body. It is obvious that this feature of the matter transport process does not correspond to the facts.

It can be shown ${ }^{2}$ on the basis of dimensional analysis that the levelling-off time of the substance concentration over the whole water body will be equal to

$$
t=\text { const } L^{2} / D
$$


where $L$ is the characteristic length of the water body, and for the diffusion velocity of the substance

$$
\omega=(c D / \tau)^{\frac{1}{2}}
$$

where $\tau$ is the relaxation time.

The relationship describing the non-instantaneous transport of substances over the whole water body can be constructed making use of the ideas of the theory of fluid rheology, especially that of viscoelastic liquids.

The rate of flow of a substance may be written for the one-dimensional problem as follows

$$
q_{x}=-D \partial S / \partial x-c\left(D / W_{x}\right) \cdot(\partial q / \partial t)
$$

If the diffusion velocity is large, equation (7) becomes equivalent to the ordinary gradient-type diffusion relationship (1).

The movement of the plume front of the substance is described by the last term of relationship (7), i.e.

or

$$
q_{x}=-c\left(D / W_{x}\right)(\partial q / \partial t)
$$

$$
q=q_{0} \exp \left(-L W_{x} / D\right)
$$

where $q_{0}$ is the rate of flow of substance at the distance $L=0$.

The corresponding one-dimensional mass balance equation becomes

$$
\frac{\partial S}{\partial t}+c \frac{D}{w^{2}} \frac{\partial^{2} S}{\partial t^{2}}+u \frac{\partial S}{\partial x}=D \frac{\partial^{2} S}{\partial x^{2}}
$$

which is hyperbolic.

The analogous three-dimensional mass balance equation may be written assuming only that the diffusion velocity is constant, and does not depend on the coordinate directions.

The corresponding transport mechanism for substances suspended in water is more complicated, although in that case the internal forces act directly on the suspended particles as well as indirectly through the water in the form of interaction between fluid and suspended components. Basic equations for the transport of suspended substances have been derived by several scientists ${ }^{3,4}$. A short review of the transport of suspended substances in a water body has been published by Vasiljev ${ }^{5}$.

\section{TRANSPORT MECHANISM IN TURBULENT FLOW}

Many papers and monographs have reviewed turbulent transport mechanisms in water ${ }^{6-9}$. Therefore we present here only a very short survey on the turbulent transport of substances.

In general, flow in natural water bodies is more or less turbulent. The water flow becomes turbulent because of local inertial forces. Turbulent flow is characterized by random changes of all its physical parameters, such as velocity, pressure, temperature, density. Turbulent flows represent, from the mechanical point of view, non-linear systems with unlimited numbers of degrees of freedom. Thus the theory of turbulence can normally be only 
statistical $^{6}$. As the flow represents a random field, then for its description one has to make use of probability characteristics - for example, of statistical momenta, probable densities and distributions, spectral densities, etc.

Usually turbulent flow is described by means of its two first statistical moments: mathematical expectation and variance. The evaluation equations of the first two moments comprise and are solved by fixed initial and boundary conditions. Only in recent years have additional evaluation equations for other statistical characteristics been used for description of turbulent flow.

Determining the mathematical expectations or, more simply, mean values of turbulent flow, one must average over the ensemble of turbulent field realizations. As a rule we do not have any ensemble of the field realizations because we cannot repeat in practice the same flow conditions. So we must make use of series of measurements of turbulent field characteristics, e.g. we assume the realizations to be ergodic ones. This assumption is generally more or less correct.

In turbulent flow the substances are transported by both molecular diffusion and turbulent motion of the water. There is thus an interaction of both transport mechanisms. The turbulent transport of substances occurs by means of the random motion of water, which can be divided into mean velocity and turbulent components

$$
\vec{V}=\overrightarrow{\vec{V}} \pm \vec{V}^{\prime}
$$

In the same way the concentration of the substance can be divided into a mean concentration and a fluctuation of the concentration:

$$
S=\bar{S} \pm S^{\prime}
$$

Turbulent flow consists of a hierarchy of vortexes of several sizes. In this hierarchy the small-size vortexes come into existence by loss of stability of the large vortexes. This process continues down to the minimum size at which the vortexes would be stable. At this size the energy of turbulence is transformed by viscosity into heat.

Thus the mean flow of energy is instituted at the largest vortexes and then transformed by a cascade process continuously from the larger vortexes to the smaller ones and so to stable vortexes.

According to the cascade model of turbulence, vortexes of different sizes take part in turbulent mass transport. Thus, the greater the plume becomes, the faster it will be transported over the whole water body.

The concentration field $S(\vec{x}, t)$ of passive substances can be described according to Monin and Jaglom ${ }^{6}$ for a very individual realization of the turbulent flow for regions without sources of substances by means of the molecular diffusion equation (4). Replacing in this molecular diffusion equation both velocity and concentration by their mean and turbulent values and averaging all terms of the equation, we get

$$
\frac{\partial \bar{S}}{\partial t}+u_{\alpha} \frac{\partial \bar{S}}{\partial x_{\alpha}}=-\frac{\partial}{\partial x_{\alpha}} \overline{u_{\alpha}^{\prime} S^{\prime}}+D \frac{\partial^{2} \bar{S}}{\partial x_{\alpha}^{2}}
$$

The new term that appears in the equation-in comparison with the molecular diffusion equation-represents the turbulent rate of mass flow 


$$
q_{\alpha}=\overline{u_{\alpha}^{\prime} S^{\prime}}
$$

Equation (12) is not of closed form, because the turbulent mass flow is unknown. The simplest way of determining the turbulent mass transport would be to make use of the analogy with the molecular mass transport.

Relating the turbulent transport to the mean flow concentration gradient, one obtains

$$
q_{\alpha}=+\overline{u_{\alpha}^{\prime} S^{\prime}}=-K_{\alpha \beta} \partial \bar{S} / \partial x_{\beta}
$$

where $\boldsymbol{K}_{\alpha \beta}$ is the tensor of the turbulent diffusion coefficients. Equation (14) leads to the parabolic type of turbulent diffusion equation.

Making use of an assumption analogous to equation (7) for molecular diffusion one may get the following relation

$$
q_{\alpha}=-\boldsymbol{K}_{\alpha \beta} \frac{\partial \bar{S}}{\partial x_{\beta}}-\tau_{\alpha \beta} \frac{\partial q_{\beta}}{\partial t}
$$

where $\tau_{\alpha \beta}$ is the tensor of some coefficients analogous to the 'relaxation time' for molecular diffusion.

Kolmogorov $^{10}$ has shown that at high values of the Reynolds number there must exist a sub-range of scales much smaller than the external flow size $L$ where the turbulence would be isotropic and homogeneous. In this sub-range the turbulence is determined by viscosity and by the rate of energy flow down the cascade of vortexes. In the steady state it will be equal to the rate of energy dissipation. In this sub-range there exists a range of scales much greater than the minimum vortex scale where the turbulence is determined only by the energy dissipation rate $\varepsilon$. This range of scales is called the locally isotropic inertial sub-range. Obukhov ${ }^{11}$ has shown that in the inertial sub-range of scales the turbulent diffusion coefficient is determined as

$$
K=c \varepsilon^{\frac{1}{3}} l^{\frac{4}{3}}
$$

where $l$ is the diffusion scale.

The turbulence of steady state motion is carried down the vortex cascade with the rate equal to the energy dissipation rate $\varepsilon$. The decay time factor of the turbulence energy $\tau$ depends on the rate of energy generation $\varepsilon$ and on the space factor $l$. On the basis of dimensional analysis one can get $^{12}$

$$
\tau \sim \varepsilon^{-\frac{1}{3}} L^{\frac{2}{3}} \sim L^{2} / K(l)
$$

The above-described transfer process for the cascade energy exists for some sub-range of three-dimensional turbulent flows. There exist in nature turbulent quasi-two-dimensional flows, e.g. turbulent flows with two space dimensions much greater than the third one (ocean turbulence at great space scales, etc.). The two-dimensional theory of turbulence has been proposed $^{13,14}$. In two-dimensional turbulence the energy flow from largescale vortexes to small-scale ones cannot exist because of the lack of expansibility of the vortex lines ${ }^{15}$. In addition, in two-dimensional turbulence there exists the cascade process of entropy flow from higher to lower wavenumbers. Thus the entropy $\eta$ is determined analogously to the energy dissipation rate $\varepsilon$ as 


$$
\eta=\frac{1}{2} \frac{\mathrm{d} \overline{\Omega^{2}}}{\mathrm{~d} t}=\frac{1}{2} \gamma \overline{\left(\frac{\mathrm{d} \Omega_{\alpha}}{\mathrm{d} x_{\beta}}+\frac{\mathrm{d} \Omega_{\beta}}{\mathrm{d} x_{\alpha}}\right)^{2}}
$$

where the $\Omega_{\alpha}$ are components of the velocity vorticity and $\gamma$ is the kinematic viscosity.

$\operatorname{Lin}^{16}$ has derived by dimensional arguments for the inertial sub-range of the two-dimensional range the following relationship for the turbulent diffusion coefficient

$$
\boldsymbol{K}=A \eta^{\frac{1}{3} \bar{\xi}^{2}}
$$

where $\left(\bar{\xi}^{2}\right)^{\frac{1}{2}}$ is the relative particle separation and $A$ is a positive constant. Substituting $\bar{\xi}^{2}=l^{2}$ one can obtain

$$
\boldsymbol{K}=A \eta^{\frac{1}{3}} l^{2}
$$

The conservation equation of variance of concentration of dissolved substances $\overline{S^{\prime 2}}$ was derived first by Csanady ${ }^{17}$. Making use of the FriedmanKeller method and assuming that the molecular diffusion equation (4) is valid for every individual realization of the turbulent flow, he obtained

$$
\frac{\partial \overline{S^{\prime 2}}}{\partial t}-\bar{u}_{\alpha} \frac{\partial \overline{S^{12}}}{\partial x_{\alpha}}=-\overline{2 \overline{u_{\alpha}^{\prime} S^{\prime}}} \frac{\partial \overline{S^{\prime 2}}}{\partial x_{\alpha}}+\frac{\partial}{\partial x_{\alpha}}\left(D \frac{\partial \overline{S^{\prime 2}}}{\partial x_{\alpha}}-\overline{u_{\alpha}^{\prime} S^{\prime 2}}\right)-\chi
$$

where $\chi$ is the dissipation rate of the concentration variance.

The physical significance of the terms of equation (22) $\operatorname{are}^{17}$ : on the lefthand side we have the local and convective change of concentration variance, the latter as induced by mean velocities; on the right, production of variance by the interaction of turbulent flux and mean concentration gradient, the divergence of the flux of variance due to molecular and turbulent diffusion and, finally, the dissipation.

The concentration variance in the marine environment was later studied by Juhat ${ }^{18}$.

The semi-empirical turbulent diffusion theory needs special assumptions to relate turbulent mass flow to the characteristics of the velocity field. Dividing the velocity and concentration in turbulent flow into orthogonal components of a Wiener-Hermite functional expansion, one can get a direct relationship connecting the substance field with velocity field parameters.

Toompuu and Juhat ${ }^{19}$ obtained for steady state turbulent diffusion the following relationship for concentration mean values

$$
\bar{u}^{2} \frac{\bar{S}(\vec{\Gamma})}{\partial x_{\alpha}}=\frac{\partial}{\partial x_{\alpha}} \int_{0}^{x_{1}} \frac{\partial \bar{S}(\vec{\Gamma})}{\partial x_{\beta}^{\prime}} B_{\alpha \beta}\left(\vec{\Gamma}, \vec{\Gamma}^{\prime}\right) \mathrm{d} x_{1}^{\prime}
$$

where $B\left(\vec{\Gamma}, \vec{\Gamma}^{\prime}\right)$ is the velocity field correlation function and $\bar{S}(\vec{\Gamma})$ is the concentration of dissolved substances at the point with the coordinates $\vec{\Gamma}$.

The level of concentration of dissolved substances in turbulent flow has been studied by Juhat et al. ${ }^{20}$.

To study the turbulent transport of substances one has to solve the semiempirical turbulent diffusion equation at fixed boundary conditions making some additional assumptions concerning the turbulent diffusion coefficient. 


\section{TRANSPORT MECHANISMS IN WATER}

In the literature there are many solutions at various boundary conditions, see for example ref. 6 .

\section{REFERENCES}

${ }^{1}$ R. P. Feynman, R. B. Leighton and M. Sands, The Feinman Lectures on Physics, Vol. I. Addison-Wesley: New York (1963).

2 A. I. Kitaigorodski, Introduction to Physics, Moscow (1973).

3. F. I. Frankle, Dokl. Akad. Nauk SSSR, 92 (3) (1953).

4 G. I. Basenblatt, Zh. Prikl. Matem. i Mechan. 17 (3) (1953).

5 O. F. Vasiljev, 'Problems of two-phase flow theory'. XIII Congr. IAHR, Vol. 5-3. 'General lectures' (1969).

6 A. S. Monin and A. M. Jaglom, Statistical Hydromechanics, Vol. I Moscow (1965).

7 A. Okubo, Tech. Rep. Johns Hopkins Univ. No. 62 (1970).

8 R. V. Ozmidov, Horizontal Diffusion and Turbulent Exchange in the Ocean Moscow (1968).

9 K. F. Bowden, Third Liége Colloquium on Ocean Hydromechanics Liége (1972).

10 A. N. Kolmogorov, CR Acad. Sci. URSS, 30 (1941).

11 A. M. Obukhov, CR Acad. Sci. URSS, 32 (No. 1) (1941).

12 A. S. Monin, Weather Prognosis as a Task of Physics. Moscow (1969).

13 G. K. Batchelor, Physics of Fluids, Suppl. II (1969).

14 R. H. Kraichnan, Physics of Fluids, 10 (No. 7) (1967).

15 G. S. Golitsyn, Introduction to Planetary Atmosphere Dynamics. Hydrometpress: Leningrad (1973).

16 Jung-Tai Lin, J. Atmos. Sci. 29 (1972).

17 G. T. Csanady, J. Atmos. Sci. 24 (1967).

18 M. K. Juhat, Trudy TPI (Tallinn Polytechnic Institute), Ser. A, No. 298 (1970).

19 A. G. Toompuu and M. K. Juhat, in press (1974).

20 M. K. Juhat, R. V. Ozmidov, V. K. Astok and A. N. Gesentsvei, Izvest. Akad. Nauk SSSR, 'Atmospheric and oceanic physics', 7 (9) (1971). 